\title{
El fútbol, la pasión que muere lentamente en Ecuador
}

\section{Soccer, the passion that dies slowly in Ecuador}

\author{
Wilson Germán Nieto Aguilar
}

\begin{abstract}
Resumen
Este trabajo de investigación tiene como fin indagar sobre los hábitos deportivos y la aceptación del producto 'campeonato ecuatoriano de fútbol profesional', entre los habitantes del Distrito Metropolitano de Quito y valles aledaños. La sustentación teórica se basa en fuentes secundarias que permiten entender el mercado del fútbol en Ecuador. La realidad del mercado se evidencia mediante un estudio de carácter cuantitativo que utiliza a la encuesta como herramienta. A partir del proceso de investigación se obtienen resultados que se desprenden de 1209 encuestas: el 11.56 \% de las personas encuestadas prefiere asistir a espectáculos deportivos en relación al fútbol, mientras que 66.50 \% califican entre 1 y 7 el interés que despierta el campeonato de fútbol en Ecuador, es así que el 'rey de los deportes' en Ecuador pierde su trono como espectáculo de masas.
\end{abstract}

\section{Palabras clave}

Fútbol, liga pro, mercado fútbol, fútbol ecuatoriano, fútbol profesional, FEF, AFNA, Ecuador

Clasificación JEL: M31

\section{Abstract}

The research work aims to investigate sports habits and the acceptance of the product 'Ecuadorian professional soccer championship', among the inhabitants of the Metropolitan District of Quito and surrounding valleys. The theoretical support is based on secondary sources that allow understanding the soccer market in Ecuador. The reality of the market is evidenced by a quantitative study using the survey as a tool. From the research process results are obtained that are derived from 1209 surveys: $11.56 \%$ of people surveyed prefer to attend sports events in relation to football, while $66.50 \%$ qualify between 1 and 7 the interest that awakens the championship of soccer in Ecuador, it is so that the 'king of sports' in Ecuador loses its throne as a mass spectacle.

\section{Keywords}

Football, liga pro, soccer market, Ecuadorian soccer, professional soccer, FEF, AFNA, Ecuador.

Classification JEL: M31 


\section{Introducción}

'El fútbol, pasión de multitudes' es una frase entramada por prensa deportiva especializada y hace referencia a la práctica recreativa y al fútbol como espectáculo. Como deporte profesional, incluye a distintos actores que son parte de una sociedad, entre los cuales se destacan los aficionados, hinchas, socios, patrocinadores y los medios de comunicación. El punto de partida para la integración de los actores mencionados es el 'marketing deportivo', el cual hace uso de la publicidad como una herramienta que permite la globalización del mensaje comercial que busca la seducción y fidelización de las personas hacia los equipos de fútbol, patrocinadores, productos y otros actores relacionados al tema de estudio.

\section{Contexto histórico del fútbol en Ecuador}

Memorias del deporte fútbol, publicación patrocinada en el año 2011 por Ministerio del Deporte en la cual se relata la historia del balompié y se afirma que:

Fueron los ingleses o las familias pudientes de nuestros países que viajaron al Reino Unido, quienes introdujeron el fútbol en Ecuador. Cuenta la historia que a finales del siglo XIX, concretamente el 23 de abril de 1899, jóvenes pudientes del puerto principal formaron el Guayaquil Sport Club para proporcionar a los socios diferentes recreaciones en los siguientes juegos: Foot Ball, Cricket, Base Ball, Lawn Tenis, botes y otros. Toda esta jerga tiene su explicación en que los jóvenes del Guayaquil Sport Club habían regresado de Europa, donde realizaban sus estudios y donde se vivía el renacimiento de los Juegos Olímpicos.

Meses después, en agosto de 1899, arribaron a Guayaquil los hermanos Juan Alfredo y Roberto Wright, -quienes también habían residido en Inglaterra y habían practicado este deporte-, para pasar luego a Lima donde eran parte del Unión Cricket Club. Fue Juan Alfredo quien trajo el primer balón
El deporte en el ámbito mundial influye en el comportamiento social, cultural y comercial, por tal razón, en el campo académico se considera una vía de estudio del comportamiento del ser humano que se vincula al deporte como espectáculo, ya sea de forma activa o pasiva, el vínculo entre el individuo y el deporte se materializa en el imaginario de una sociedad (Luna-Arocas, 2003). La conducta del individuo como consumidor es influida por signos y símbolos (marcas) que son parte distintiva de una cultura y los productos o servicios que representan a las marcas se convierten en simbolos deportivos referentes de una sociedad o individuo que toman las marcas como propias y generan lealtad.

y los reglamentos de un deporte conocido como Foot Ball. (Ramírez, 2001, p. 15)

El origen del fútbol y la evolución en el Ecuador nos presentan una pauta para determinar el escenario actual de la Federación Ecuatoriana de Fútbol (FEF). Sus agremiados han disputado 60 campeonatos nacionales en la 'serie A' del fútbol profesional. La primera división del balompié ecuatoriano se dividía en serie A y B, en cada serie participan 12 equipos con sus respectivas categorías infanto-juveniles, los equipos del fútbol profesional se encuentran agremiados en 22 asociaciones provinciales que realizan la actividad deportiva, 17 escenarios son la infraestructura nacional para la práctica del deporte (FEF, 2019). El 11 de abril de 2018 se firma un convenio entre los dirigentes del fútbol ecuatoriano y los representantes de la Liga española, el fin es dar impulso a la naciente Liga Profesional de Fútbol (Bendito Fútbol, 2014), el certamen contará con 16 clubs y se disputarán 254 encuentros que 
se dividen en un todos contra todos y con la posterior clasificación a un playoffs (El Universo, 2019).

El producto fútbol en Ecuador en las decadas del 70 y 80 era exitoso y la asistencia a los estadios era masiva. En el presente, los partidos de fútbol se juegan en estadios sin público, así se confirma en la versión digital del diario El Universo (2018), que el campeón Liga de Quito convocó a 222702 aficionados al estadio Rodrigo Paz Delgado en todo el año 2018, el estadio en mención tiene una capacidad de 41575 espectadores (FEF, 2019), situación que implica que el estadio habría alcanzado su capacidad máxima en 5 oportunidades de un total de 23 fechas considerando la final del campeonato, las 17 fechas restantes se habría jugado sin público.

Al no existir 'un' modelo exitoso, corresponde a cada club elegir el propio. Su fortaleza dependerá, entre otros elementos, de la capacidad de gestión, la que a su vez está condicionada por la calificación de los ad- ministradores. Es el momento adecuado para que personas especializadas y conocedoras de un deporte complejo se incorporen a las plantas administrativas de los clubes. (Carrión y Samaniego, 2013, p. 109)

La Liga Profesional Ecuatoriana para el año 2019 diseña las reglas del campeonato, la normativa vigente permite normalizar y controlar a los actores directos como: jugadores, árbitros, directivos e instituciones agremiadas e, incluso, a patrocinadores y medios de comunicación especializados.

La realidad económica de los clubs de fútbol en Ecuador es similar a la del año 2018, pese a los movimientos comerciales que ejecutan los directivos, se evidencia que existen obligaciones impagas a jugadores, entrenadores y personal administrativo y el déficit de los clubs es alto frente a los ingresos que se manejan con base en la recaudación por taquillas, por ejemplo, el cotejo entre LDU y Olmedo recaudó 45754 USD (Bendito Fútbol, 2014).

\section{Comportamiento del consumidor y mercado}

El consumidor y su comportamiento tienen relación directa con el marketing y el desarrollo de un estudio con carácter científico es prioritario para entender los factores que mueven al consumidor sobre las necesidades y deseos. Según la RAE (2017), en la acepción tres se describe que necesidad es una carencia para la conservación de la vida, mientras que deseo en la acepción uno se determina como el movimiento afectivo hacia algo que se apetece, en mercados globales no es rentable que un producto depende de la necesidad para el consumo, las empresas buscan mover sus productos en función de elementos intrínsecos como la marca o el servicio posventa (Kotler y Lane, 2012).

La evolución del deporte amateur al profesional incluye una serie de acciones que han llevado a fusionar el marketing con el deporte, es así que nace el marketing de- portivo, el cual se apoya en acciones comerciales, sociales, culturales e, incluso, de mecenazgo dentro y fuera de un club deportivo, desde la perspectiva del autor: el marketing deportivo se encuentra ligado a la teoría de David Mcclelland cuya teoría se basa en la necesidad adquirida y moldeada a lo largo de la vida. La motivación que mueve a los individuos se sustenta en tres pilares: logro, poder y afiliación (Aiteco Consultores, 2019).

En el caso del balompié los motivadores descritos se aplican a los clubs de fútbol y a los estamentos reguladores, además engloba a los aficionados, hinchas y socios.

La necesidad de poder, en el caso del fútbol, se centra en logros deportivos y la rentabilidad económica, la necesidad de afiliación se determina como: aficionado, hincha o socio, la relación directa de estos con el club ocurre por el espectáculo o por el sen- 
tido de pertinencia que se personifica en los signos, símbolos y colores que representan al club, la necesidad de logros, en el fútbol, se reflejan en la conquista de campeonatos, que son motivo de vanidad de todos quienes son parte del equipo campeón.

El poder se genera en base a réditos económicos, resultado de la comercialización de productos o servicios ofertados por la institución. Para alcanzar el poder económico, es prioritario el análisis del comportamiento del consumidor, el mismo que se centra en el análisis de los procesos que ejecuta una persona al momento que identifica su necesidad o deseo y partiendo de ellas se desarrolla un proceso de compra. La influencia del comportamiento de un individuo se basa en factores sociales, económicos, culturales, políticos y deportivos del grupo objetivo, el fin del análisis es identificar hábitos de compra y uso de un producto o servicio, los resultados aportan en la parametrización de comportamientos que en lo posterior permitan diseñar producto tangibles o intangibles que cumplan las expectativas e incrementen la demandan los distintos nichos de mercado. sobre:

Kotler y Armstrong (2012), explican

El modelo básico del comportamiento de compra del consumidor es el modelo de estímulo-respuesta, según el cual los estímulos de marketing (las cuatro P) y otras fuerzas importantes (económicas, tecnológicas, políticas, culturales) ingresan en la 'caja negra' del consumidor y originan ciertas respuestas. Una vez dentro, producen respuestas observables en el consumidor, como la elección del producto, la elección de la marca, el momento de compra y la magnitud de la compra. (p. 158)

En esencia, uno de los propósitos del marketing busca crear lasos o vínculos entre la empresa, producto, marca y los consumidores; para conseguir este tipo de relación redituable se establecen cinco enfoques o conceptos que tienen una orientación hacia la produc- ción, producto, ventas, marketing y marketing social, en el caso del fútbol se considera que el concepto que aplica es en relación al producto; "la idea de que los consumidores prefieren los productos que ofrecen lo mejor en cuanto a calidad, desempeño y características, y que, por lo tanto, la organización debería dedicar su energía a mejorar continuamente sus productos" (Kotler y Armstrong, 2012, p. 9).

El marketing deportivo es un término que está de moda, pero ¿qué es? Gottfried (2011), propone la siguiente definición:

El marketing deportivo, como ya se ha definido es ese conjunto de estrategias y acciones que tienen como fin la comercialización del deporte en dos esferas, la primera supliendo las necesidades de los consumidores y la segunda utilizando el deporte como instrumento de comercialización de otro tipo de productos y/o servicios. Para lograr este fin utiliza dos estrategias fundamentales: el mecenazgo y el patrocinio. (p. 13)

Con el fin de enriquecer el tema de estudio, el autor define marketing deportivo como: el proceso comercial y social donde una institución deportiva o relacionada con el deporte aplica estrategias que se enfocan en el posicionamiento de las marcas, productos o servicios que oferta la empresa y así alcanzar la fidelización del público objetivo optimizando los recursos humanos, tecnológicos y económicos disponibles en la empresa con el único fin de volverse competitivos ante el mercado.

Tomando en consideración aspectos de la composición de una sociedad, el proveedor del producto o servicio deberá "tomar decisiones relativas a su mercado meta, surtido de productos y servicios, precios, promoción y plaza (ubicación). Los mayoristas de más éxito son los que adaptan sus servicios a las necesidades de sus proveedores y de sus clientes" (Kotler y Lane, 2012, p. 470).

Las estrategias en relación a las ' $4 \mathrm{p}$ de marketing' son fundamentales para la 
ejecución del marketing deportivo, además es conveniente tener presente como objeti-

\section{Objetivo}

Investigar los hábitos deportivos y la aceptación del producto 'campeonato ecuatoriano de

\section{Metodología}

La metodología empleada para el levantamiento de la información está descrita en el libro Estudio de Mercado del fútbol ecuatoriano, capítulo Pichincha (Detrás del juego, 2018).

El levantamiento de la información se realiza mediante encuestas como herramienta del método cuantitativo y el fin del instrumento es recolectar información para conocer el comportamiento comercial de los individuos además de los hábitos, costum- vo la penetración de nuevos mercados y la comunicación comercial.

fútbol profesional', entre los habitantes del Distrito Metropolitano de Quito y valles aledaños.

bres y actitudes, es así que la investigación desde la academia sustenta los procesos desde el análisis teórico en fuentes bibliográficas hacia la investigación descriptiva.

Los resultados conseguidos a través del proceso metodológico cuantitativo se desprenden de la aplicación y análisis de 1209 encuestas válidas distribuidas en Quito y valles aledaños, entre un grupo de hombres y mujeres cuya edad comprende entre 18 a 50 años.

Tabla 1. Ficha técnica

\begin{tabular}{|c|c|}
\hline UNIVERSO & Hombres y mujeres aficionados al fútbol \\
\hline TAMAÑO MUESTRA & 1214 entrevistas físicas \\
\hline MÉTODO DE MUESTREO & $\begin{array}{l}\cdot \text { Muestreo aleatorio simple por cuotas } \\
\text { ZONAS GEOGRÁFICAS: Pichincha, (Cantones Quito y Rumiñahui) } \\
\cdot \text { HÁBITAT : } 7 \text { zonas } 26 \text { puntos de levantamiento de la información } \\
\cdot \text { EDAD: } 18 \text { - 50, más. } \\
\text { - OCUPACIÓN: indistinta }\end{array}$ \\
\hline TIPO DE ENCUESTA & Personal \\
\hline $\begin{array}{l}\text { HERRAMIENTA } \\
\text { TIPOS DE VARIABLE } \\
\text { INFORMACIÓN } \\
\text { TRABAJO DE CAMPO } \\
\end{array}$ & $\begin{array}{l}42 \text { variables } \\
\text { Cualitativas (nominales / ordinales) } \\
\text { Perfil del encuestado, afiliación deportiva, consumo producto fútbol, } \\
\text { Entrevistadores personales. }\end{array}$ \\
\hline \multirow{3}{*}{ MUESTRA } & $\mathrm{K}=2\left(\sqrt[2]{\frac{p(1-p)}{n}}\right) \quad \mathrm{K}=2\left(\sqrt[2]{\frac{0,5(1-0,5)}{1.214}}\right) \quad \mathrm{K}=2\left(\sqrt[2]{\frac{0,5 \times 0,5}{1.214}}\right) \quad \mathrm{K}=2\left(\sqrt[2]{\frac{0,25}{1.214}}\right)$ \\
\hline & $\mathrm{K}=2(\sqrt[2]{0,000206}) \quad \mathrm{K}=2 * 0,014350289 \quad \mathrm{~K}=0,0287 * 100=2,87$ \\
\hline & $n=\frac{4 p(1-p)}{R^{2}} \quad n=\frac{4 * 0,5(1-0,5)}{2,87,2} \quad n=\frac{4 * 0,5(0,5)}{2,87^{2}} \quad n=\frac{2(0,5)}{2,87^{2}} \quad n=\frac{1}{0,000824} \quad n=1.214$ \\
\hline
\end{tabular}


El trabajo de campo se realizó entre los días 28 de noviembre hasta el 11 de diciembre de 2017. Considerando las zonas con mayor densidad poblacional, se establece como puntos de contacto los centros comerciales que se encuentran dispersos en distintos límites geográficos. La selección de los puntos para levantar la información se fundamentada en que estos centros comerciales acogen a una población flotante de diversos barrios de la ciudad, además de otras características sociales, comerciales, políticas o culturales.

El instrumento se compone de 42 preguntas con formato cualitativo (nominales y ordinales), el banco de preguntas se diseña varias secciones:

En la primera sección las preguntas aportan con información sobre aspectos demográficos, geográficos y psicográficos, en relación a construir el perfil del consumidor ecuatoriano. La segunda sección, busca identificar los hábitos deportivos de los individuos que son parte de la muestra. La ter-

\section{Resultados}

El fútbol en cuanto deporte en ocasiones se convierte en el centro de atención de la sociedad y genera toda clase de debates, las polémicas que se generan en torno al deporte sobrepasan la barrera de lo deportivo y se propagan al campo administrativo, político, legal y económico. En el caso ecuatoriano, el fútbol ha tenido avances en la consecución de logros deportivos como selección, clubs de fútbol, además de la internacionalización de jugadores ecuatorianos así como también grandes escándalos de corrupción deportiva. Los casos más sonados son: trata de personas y lavado de activos que implicó a exfuncionarios de la FEF (Hora 25, 2018). cera sección incluye una serie de preguntas sobre el consumo del producto fútbol como espectáculo. La cuarta sección considera preguntas que aporten al conocimiento sobre la percepción que tiene el ecuatoriano en relación con la violencia en el fútbol. La quinta sección hace un compendio de preguntas para conocer sobre el fútbol como producto en Ecuador y la sexta y última sección se diseña con una serie de preguntas para identificar la influencia de los líderes de opinión en prensa deportiva y la preferencia de consumo en medios de comunicación.

Los datos que se obtienen del trabajo de campo a través de la encuesta son transcritos a una base en una hoja de cálculo Excel con el fin de realizar una depuración de los contenidos y para expresar los resultados en tablas estadísticas se emplea el software Dyane 4.0. Con los resultados que arroja la investigación se pretende dar respuesta a la pregunta de investigación ¿el producto fútbol en el Ecuador es rentable?

En el momento que se planteó la investigación se consideró que para ser parte de la muestra se debía encontrar un equilibrio sobre los porcentajes de participación de hombres y mujeres, el resultado de la investigación nos arroja que el $60.13 \%$ son hombres mientras que el $39.87 \%$ son mujeres. La participación de las mujeres es más baja debido a que se muestran renuentes a contestar encuestas sobre el tema deportes (fútbol), mientras que la participación de los hombres es más alta y se encontraron más abiertos a responder la encuesta. 
Figura 1. Gráfica del género de los participantes del estudio

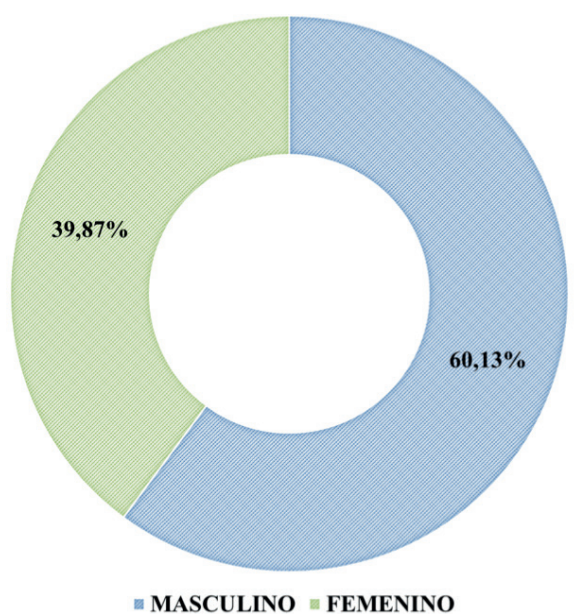

Fuente: Elaboración propia

Figura 2. Cruce de la edad con el género

Q HOMBRES MUJERES

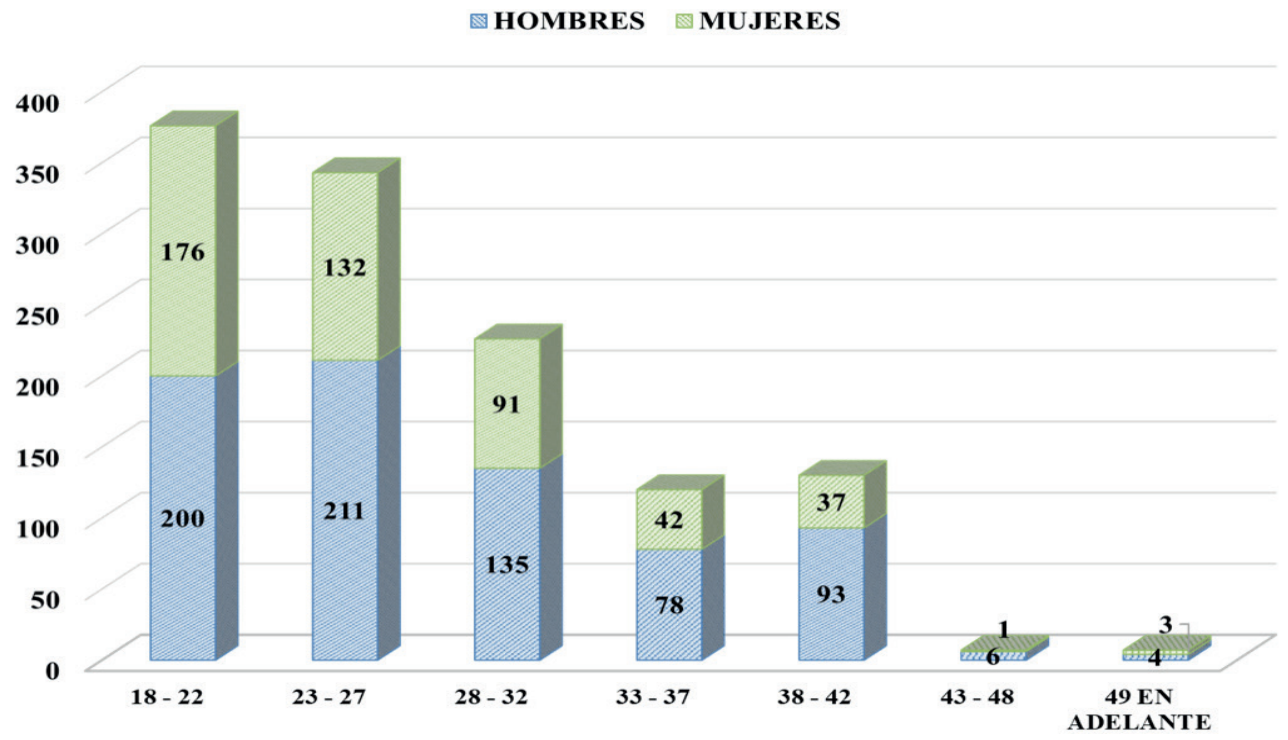

Fuente: Elaboración propia

Se consideraron como parte de la muestra personas que tienen entre los 18 años en adelante, no se considera como parte del estudio a menores de edad debido a la siguiente condicionante:

Los menores de edad tienen derechos y obligaciones y están amparados bajo el
Código de la Niñez y Adolescencia, razón que implica permisos de sus tutores legales para ser parte del estudio.

La muestra se compone de hombres entre 18 a 42 años que representan el 98.84 $\%$ del total de la muestra, otro de los factores a considerar es que este grupo de edad 
es parte de la población económicamente activa del Ecuador; lo que implica que están en la capacidad de generar recursos mone- tarios y asignarlos en actividades acorde a sus hábitos de vida y comportamientos de compra.

Figura 3. Percepción del deporte que genera violencia entre los asistentes

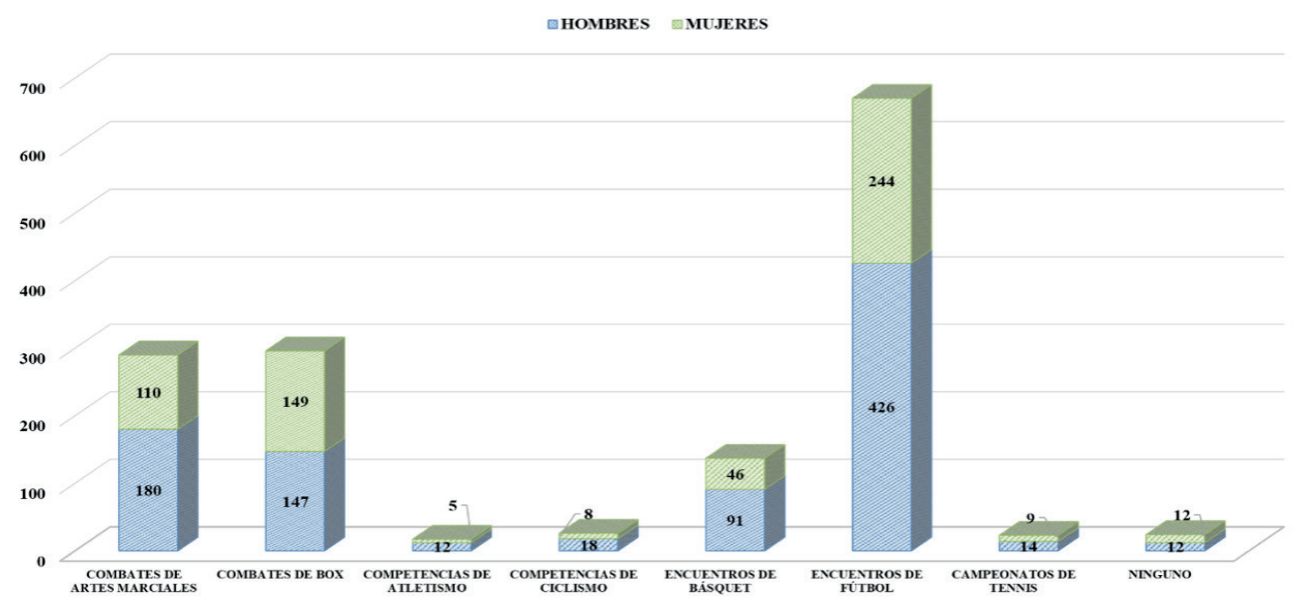

Fuente: Elaboración propia

Los distintos actores relacionados con el deporte fútbol, plantean la hipótesis que la violencia en los estadios es una de las causas para que los aficionados no asistan al fútbol y así lo corrobora la pregunta que se planteó para conocer la percepción del encuestado sobre el deporte que genera violencia, la respuesta es del $45.18 \%$ de personas que ratifican que el espectáculo del fútbol es generador de violencia y así lo describe el articulo del Diario El Comercio del 7 de febrero de 2017, en el cual se citan quince actos de violencia relacionados con las barras de los equipos del fútbol ecuatoriano (Puruncajas, 2017).

Figura 4. Cruce sobre la asistencia a partidos de fútbol con género

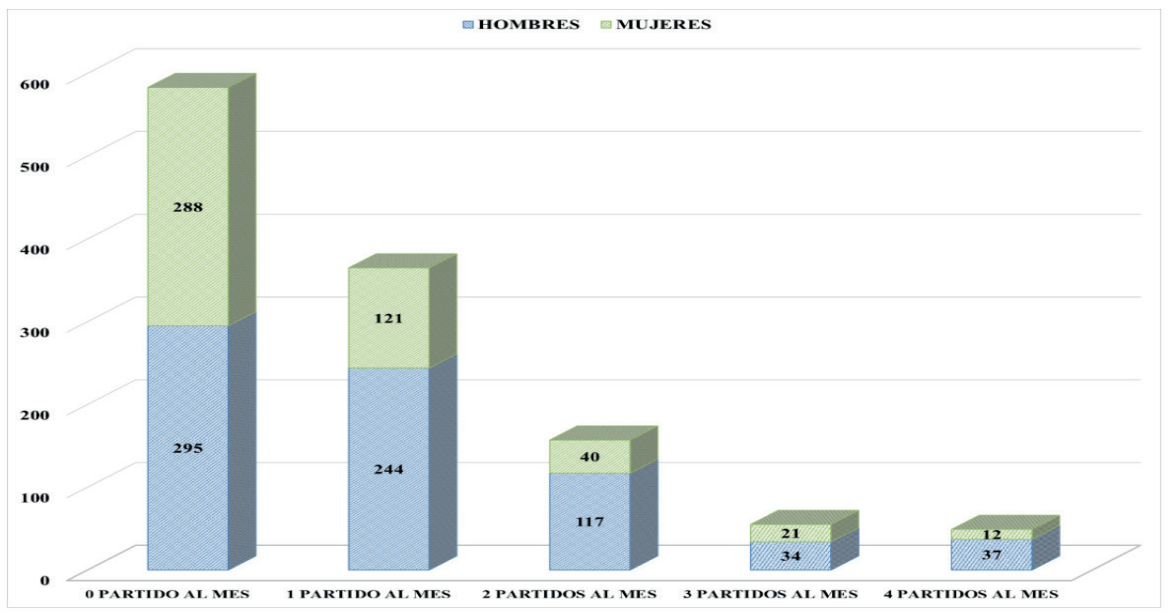


El $48.22 \%$ de personas sobre el total de la muestra investigada responde que no asiste a encuentros de fútbol y tan solo el
$30.19 \%$ afirma asistir a un encuentro de fútbol al mes.

Figura 5. Cruce sobre la asistencia a espectáculos o actividades de ocio con género

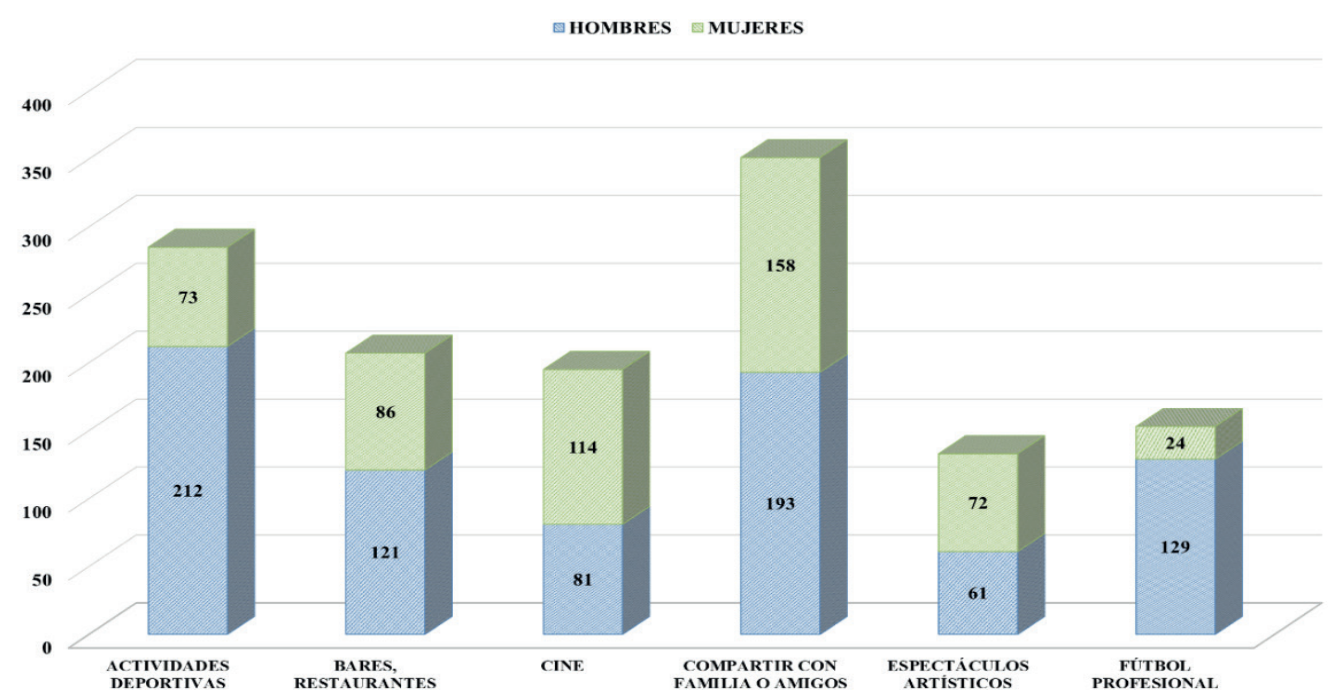

Fuente: Elaboración propia

El $11.52 \%$ afirma un interés por la asistencia a mirar encuentros de fútbol del campeonato ecuatoriano. El resto de encuestados prefirieren realizar actividades de otra índole como el compartir con amigos y familia en un $26.51 \%$ o realizar actividades deportivas en un $21.53 \%$.

Figura 6. Cruce sobre el interés del fútbol como espectáculo con género

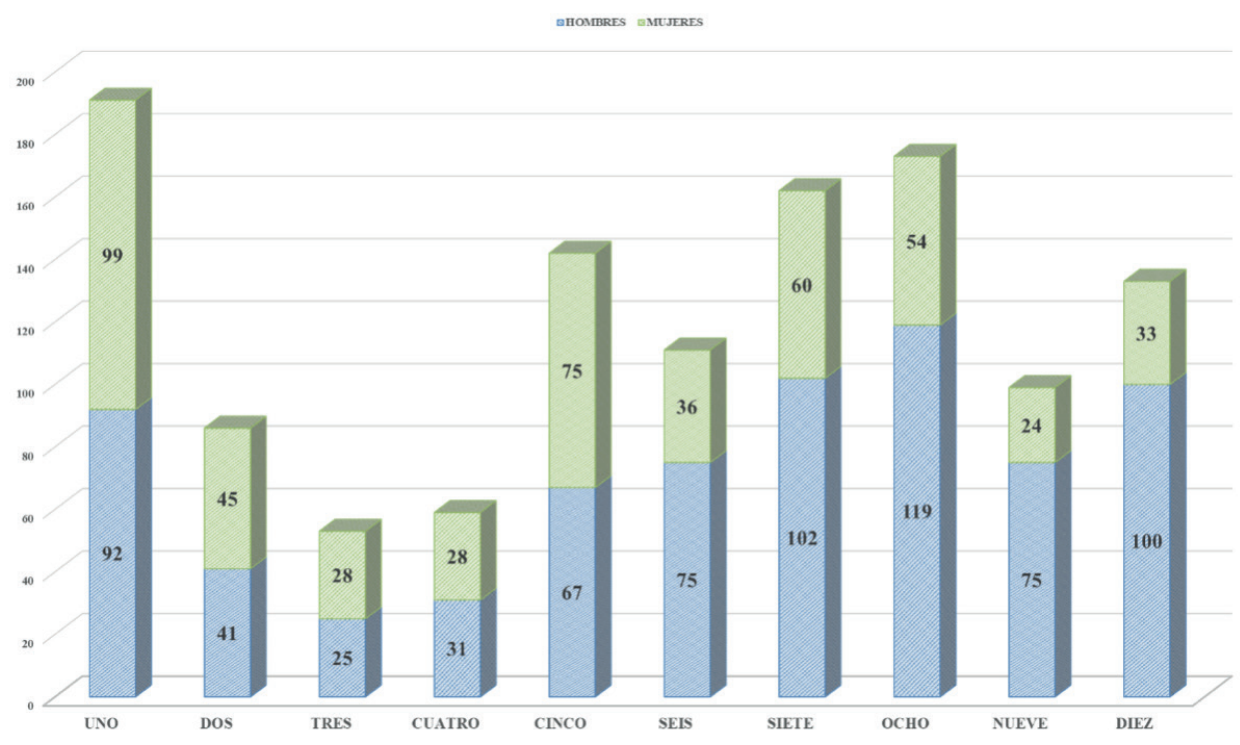

Fuente: Elaboración propia 
El $56.10 \%$ de los encuestados califica el fútbol ecuatoriano como un producto deficiente y asigna una calificación entre uno y seis. Como producto compite con otro tipo de espectáculos, además de la sobre oferta por televisión.

Figura 7. Cruce sobre el posible precio de las entradas al fútbol profesional con género

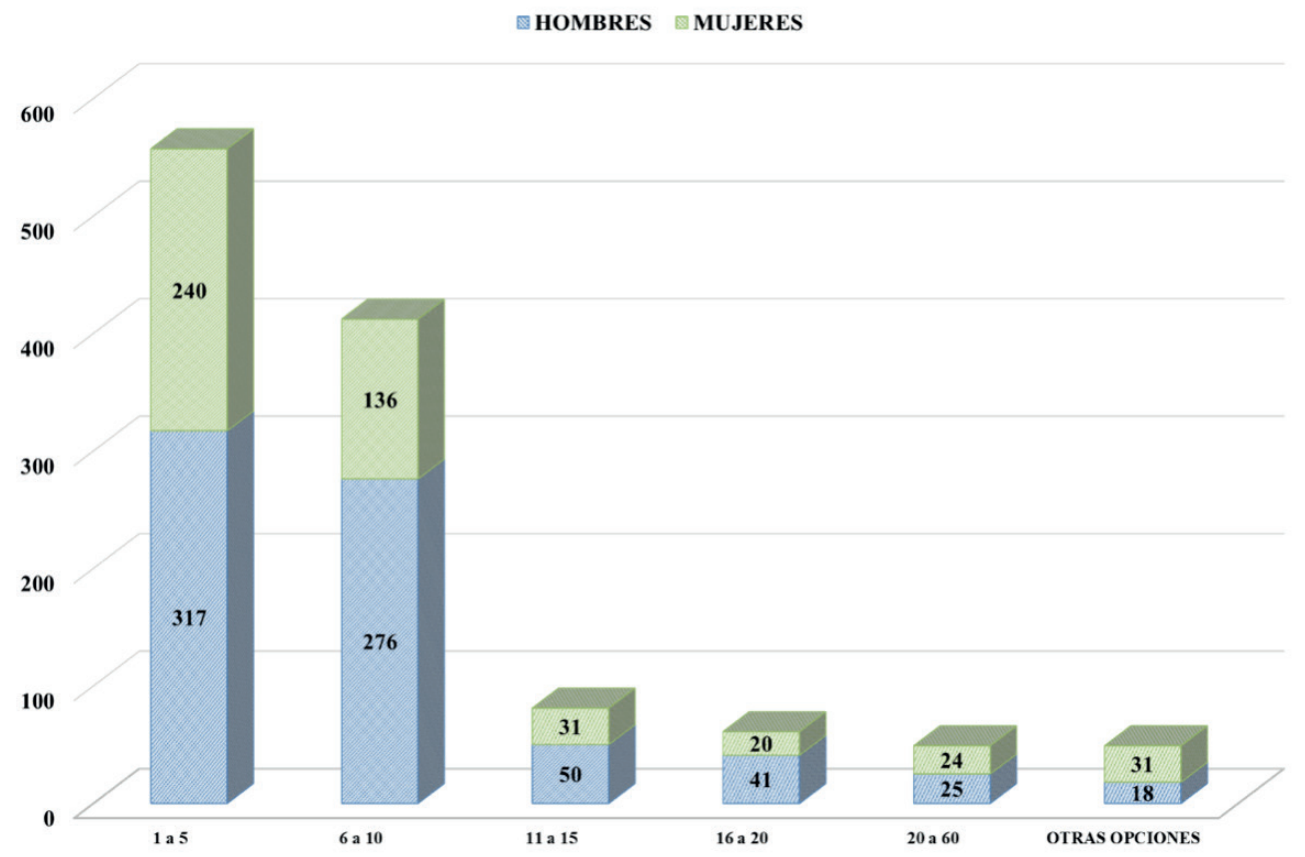

Fuente: Elaboración propia

El precio de las entradas es un factor determinante para la asistencia a un espectáculo, la variable precio en marketing cumple con la función de discriminar entre públicos objetivos. Por esta razón, los estadios se dividen por localidades que suelen ser denominadas como: general o popular, preferencia, tribuna y palco. En la encuesta se

\section{Conclusión}

El fútbol es un producto rentable que genera millones de dólares e involucra una serie de actividades directas e indirectas que contribuyen a reforzar la industria a nivel mundial. La Federación Ecuatoriana de Fútbol y los clubs asociados, no han sido capaces de consolidar de forma comercial un producto que en otros países es rentable $y$, al contrario, se consultó sobre el valor que los encuestados estarían dispuestos a pagar por asistir a un partido de fútbol y la respuesta varía entre 1.00 a 5.00 USD de un total de $46.07 \%$. La moda estadística es de 3.00 USD, además es importante resaltar que un $3.64 \%$ afirma que no estaría dispuesto a pagar por presenciar partidos de fútbol en Ecuador.

muestran impávidos dejando que la violencia cobre vidas dentro y fuera de los estadios.

El marketing, es una bandera que flamea en épocas de campaña electoral, queda en el papel debido a que no existen acciones comerciales planificadas que perduren en el tiempo y sean un aporte directo al presupuesto general de los clubs. 
La FEF y los clubs ecuatorianos no asumen que es prioritario el conocimiento de la sociedad y los públicos objetivos en relación al comportamiento social, comercial y deportivo. Para entender estos hábitos y comportamientos es fundamental desarrollar estudios de investigación de mercados los cuales pueden ser de carácter cualitativo o cuantitativo, estos estudios deberían ser planteados de forma individual por los clubs y en función de los resultados deberán tomar decisiones que aporten al diseño de productos y servicios atractivos para los aficionados, hinchas y socios de cada institución, mientras que la Federación Ecuatoriana de Fútbol debería plantear estudios nacionales pesando en aspectos como:

- Aceptación de marcas para incluirlas como auspiciantes

- Aceptación del producto fútbol

- Modelos de campeonato

- Precios de las entradas

- Servicios directos e indirectos para mejorar la asistencia al fútbol

- Televisión paga

- Otros aspectos comerciales

No solo los aspectos comerciales son prioritarios de estudio en el fútbol también los sociales, culturales y deportivos en relación con los actores directos (futbolistas), las entrevistas que realiza la gente especializada de la prensa deportiva evidencian una realidad social, cultural y económica de los futbolistas que en algunos casos el jugador llega a ser cabeza de toda una familia y el sueldo de este se convierte en el único sustento familiar.

En Ecuador si no se sientan las bases para el desarrollo de estudios del comportamiento del consumidor actual y futuro, el declive y muerte del fútbol es inminente, se evidencia que encuentros de fútbol se celebran tan solo con el $1 \%$ del total del público que puede ingresar en un escenario, el estu- dio que se presenta a consideración del lector, arrojan los siguientes resultados:

- El $56.08 \%$ de la muestra afirma haber presenciado o participado en riñas o enfrentamiento entre aficionados de dos clubs.

- La percepción de la hinchada más violenta del Ecuador es la del Barcelona S. C., con un $71.13 \%$, seguida por las barras del club Emelec y de Liga Deportiva Universitaria con un $11.50 \%$ y $10.01 \%$ respectivamente.

- La violencia en el fútbol es atribuido a las barras bravas en un $70.74 \%$.

- El $67.33 \%$ del total de la muestra afirma que suele mirar partidos de fútbol por televisión paga, se asume que el valor de las entradas se destina al pago de la mensualidad de cable.

- El 51.20 \% considera que el modelo de campeonato actual no es atractivo y expresan que se debería jugar por año dos campeonatos cortos.

- Los ingresos que reciben los futbolistas vs el espectáculo que brindan no están acorde, según lo expresa el $59.22 \%$; mientras que el $54.01 \%$ considera que los futbolistas deberían recibir sus ingresos por partido ganado.

Los resultados presentados son parte de un cuestionario que incorporó 42 preguntas que evidencian la realidad que atraviesa el fútbol profesional en Ecuador, este estudio debe ser tomado como el punto de partida para futuras investigaciones que lleven a entender el comportamiento del consumidor en relación al deporte y a los espectáculos de índole comercial.

Los resultados que se obtienen como parte del trabajo de investigación evidencian la agonía del fútbol profesional en Ecuador e invitan a la reflexión de las actividades deportivas y comerciales relacionadas con el deporte, debido a que el producto fútbol 
profesional no es rentable en Ecuador y va perdiendo espacio frente a otro tipo de espectáculos y actividades.

\section{Referencias}

Aiteco Consultores, SL. (2019). Recuperado el 11 de marzo de 2019 de https://www.aiteco. com/mcclelland-teoria-necesidades/.

Bendito Fútbol. (2014). Bendito Fútbol. Recuperado el 8 de Marzo de 2019, de https://www. benditofutbol.com/nacional/campeonatoecuatoriano-liga-profesional-firma-convenio.html.

Carrión, F., y Samaniego, P. (noviembre-diciembre de 2013). La crisis del fútbol ecuatoriano. Entre el endeudamiento, la fragilidad institucional y la violencia. Nueva Sociedad, 109. Recuperado el 20 de agosto de 2017.

El Universo. (2016 de octubre de 2016). Barcelona SC no envió a Emelec a la serie B. Guayaquil, Guayas. Recuperado de http://www. eluniverso.com/deportes/2016/10/30/ nota/5880685/barcelona-no-envio-emelec-serie-b.

El Universo. (20 de diciembre de 2018). Emelec fue el equipo más taquillero de 2018. Recuperado el 22 de marzo de 2019 de https://www. eluniverso.com/deportes/2018/12/20/ nota/7106625/emelec-domino-asistencia-publico-recaudacion-local-2018.

El Universo. (2019). Liga profesional 2019, 16 equipos que deberían descender. Recuperado el 11 de marzo de 2019 de https://www.eluniverso.com/deportes/2018/08/21/nota/6916583/liga-profesional-2019-16-equipos-incluidos-quedeberian-descender.

Federación Ecuatoriana de Fútbol. (19 de septiembre de 2017). FEF. Recuperado de Organización Estadios: http://ecuafutbol. org/web/estadios.php.

Federación Ecuatoriana de Fútbol. (14 de septiembre de 2017). Ecuafutbol. En Historia de la Federación Ecuatorina de Fútbol. Recuperado de http://www.ecuafutbol.org/ web/historia.php.
Federación Internacional de Fútbol Asociado (FIFA). (2017). FIFA MAGAZINE. Retrieved from GRAN CENSO 2016. Recuperado de http://es.fifa.com/search/index. htmx?q=practica+del+futbol.

Gottfried, V. J. (2011). Nuevas tendencias y avances en el marketing deportivo en Colombia. Universidad Militar Nueva Granada, 1-33.

Hora 25. (5 de noviembre de 2018). La crisis del fútbol ecuatoriano. Teleamazonas. Recuperado el 13 de marzo de 2019 de http:// www.teleamazonas.com/hora25ec/lacrisis-del-futbol-ecuatoriano/.

Instituto Nacional de Estadística y Censo. (2008). Composición de los hogares ecuatorianos. Quito: INEC.

Instituto Nacional de Estadísticas y Censos. (30 de junio de 2017). Ecuador en Cifras. En Proyección de la población del Ecuador por edades. Obtenido de http://www. ecuadorencifras.gob.ec.

Koontz, H., Weihrich, H., y Cannice, M. (2012). Administración. Una perspectiva golbal y empresarial. CDMX, México: McGraw-Hill.

Kotler, P., y Armstrong, G. (2012). Marketing. CDMX, México: Pearson Educación.

Kotler, P., y Keller, K. (2012). Dirección de Marketing. CDMX, México: Pearson Educación.

Kotler, P., y Lane, K. K. (2012). Dirección de Marketing. CDMX, México: Pearson Educación.

Luna-Arocas, R. (2003). Segmentación psicográfica y marketing deportivo. Revista de psicología del deporte, 122.

Nieto Aguilar, W. G., Luna Montalvo, A. A., y García Moreno, J. G. (2018). Detrás del juego. Quito: UTE.

Nogales, Á. F. (2004). Investigación y técnicas de mercados. Madrid: ESIC.

Puruncajas, Á. (2017 de febrero de 2017). Deportes. Los actos de violencia en el fútbol ecuatoriano en una década.

RAE. (18 de septiembre de 2017). Real Academia Española. Recuperado de http://www.rae.es/.

Ramírez, J. (2001). Ecuador: País futbolero. Quito: OCHOYMEDIO. 